\title{
Born-Oppenheimer approximation in open systems
}

\author{
X. L. Huang and X. X. Yi* \\ School of Physics and Optoelectronic Technology, \\ Dalian University of Technology, Dalian 116024 China
}

(Dated: May 30, 2018)

\begin{abstract}
We generalize the standard Born-Oppenheimer approximation to the case of open quantum systems. We define the zeroth order Born-Oppenheimer approximation for an open quantum system as the regime in which its effective Hamiltonian can be diagonalized with fixed slowly changing variables. We then establish validity and invalidity conditions for this approximation for two types of dissipations - the spin relaxation and the dissipation of center-of-mass motion. As an example, the Born-Oppenheimer approximation for a two-level open system is analyzed.

PACS numbers: 03.65.Bz, 07.60.Ly
\end{abstract}

\section{INTRODUCTION}

Schrödinger equation which governs the dynamics of quantum system is among the fundamental equations in physics. In principle we can predict all features of a quantum system at zero temperature by solving this equation. However, many problems are hard to handle and the Schrödinger equation might not be solved analytically. Many approximations have been developed in order to solve this problem, including the adiabatic approximation [1] and the Born-Oppenheimer(BO) approximation [2].

The adiabatic approximation tells us that for a sufficiently slowly varying Hamiltonian, if the system initially is in an instantaneous eigenstate of the Hamiltonian, the system will remain in that eigenstate up to a phase factor at later times (for the adiabatic condition and recent

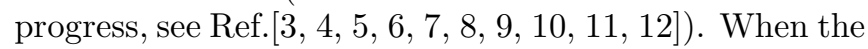
quantum system evolves adiabatically and cyclically, the eigenstate of the Hamiltonian will acquire a Berry phase [13] except the dynamical phase. This concept was generalized to non-cyclic and non-adiabatic cases, and the non-dynamical phase acquired in this situation was called the geometric phase [14, 15, 16, 17, 18, 19, 20, 21]. If a quantum system has two sets of variables, say a fast and a slow set of variables, the BO approximation can be used to solve the dynamics, this can be done by solving the fast variables with fixed slow variables first, leading to an effective Hamiltonian for the slow variables. Applying this approximation to a bipartite particle, an external vector potential is introduced for the slowly moving particle due to its fast moving partner [22]. Thus the total wave-function can be factorized to a product of two wave-functions corresponding to the fast and the slow variables. The $\mathrm{BO}$ approximation has been widely used in many fields of physics and chemistry and proved to be a fundamental tool in these research [23].

These two approximations are both for closed quantum systems. However, due to the unavoided couplings

*yixx@dlut.edu.cn of quantum system to its environment, a realistic quantum system is in general open and the dynamics of such a system can be described by the so-call Markovian master equation. The master equation was derived from Schrödinger equation by tracing out the environment variables in the Markovian limit $|24|$ (for the solution of this equation, see e.g. Ref. [25, 26, 27, [28, 29, 30] and for the dynamics beyond the Markovian limitation, see Ref. 32, 33, 34, 35, 36, 37, 38, 39, 40]). The adiabatic approximation has been extended to the case of open systems by several authors [41, 42, 43, 44, 45, 46, 47]. One of these extensions was done by the effective Hamiltonian approach [45, 46, 47]. The key idea of the effective Hamiltonian approach can be outlined as follows. By introducing an ancilla which is the same as the quantum system, we can map the density matrix of the quantum system to a wave-function of the composite system (system plus ancilla). Then the master equation can be mapped to a Schrödinger-like equation. Since the dynamics of open systems is governed by a Schrödinger-like equation, some conclusions for the closed system can be extended to open systems straightforwardly. In this paper, we extend the BO approximation to open systems by using the effective Hamiltonian approach. Two types of dissipation, the spin relaxation and the dissipation in the center-of-mass motion(DissCOM), will be considered. The validity condition for the $\mathrm{BO}$ approximation is also presented.

The structure of this paper is organized as follow. In Sec II we generalize the BO approximation to open systems with spin relaxations. The case of dissipation in the center-of-mass motion (DissCOM) is considered in SecIII In SecIV we present an example for the case of spin relaxation to show the details of the formulism. Conclusions and discussions are given in Sec $\mathrm{V}$

\section{SPIN RELAXATION}

Consider a spin moving in a magnetic field. Taking the spin relaxation into account, the evolution of the density matrix for such a system can be described by the Markovian master equation that generally can be written in the 
following form $(\hbar=1)$ 24],

$$
\frac{\partial}{\partial t} \rho=-i[H, \rho]+\mathcal{L} \rho,
$$

where $H$ is the Hamiltonian for the system. In our discussion, this Hamiltonian can be divided into the following three parts

$$
H=H_{K}+V+H_{S}(\vec{B}(\vec{x}), \vec{S}),
$$

where $H_{K}=H_{K}(\vec{x})=\frac{\vec{p}^{2}}{2 M}$ is the kinetic energy, $\vec{p}$ is the momentum operator, $V=V(\vec{x})$ denotes an external potential and $H_{S}(\vec{B}(\vec{x}), \vec{S})$ represents the coupling of the spin to an external magnetic field $\vec{B}(\vec{x})$. Note that the coupling depends on the coordinate $\vec{x}$ through the inhomogeneous external field $\vec{B}(\vec{x})$. The second term on the right hand side of Eq.(11) denotes the spin relaxation/dissipation, which is assumed here to takes the Lindblad form [48],

$$
\mathcal{L} \rho=\frac{1}{2} \gamma\left(2 \sigma^{-} \rho \sigma^{+}-\rho \sigma^{+} \sigma^{-}-\sigma^{+} \sigma^{-} \rho\right),
$$

where $\sigma^{+}$and $\sigma^{-}$are Pauli operators. For the case of DissCOM, this term is different 24]. We shall discuss this problem in Sec III.

Following the standard procedure of the effective Hamiltonian approach [31, 45, 47], we can map the density matrix into a wave-function by introducing an ancillary system, and obtain a Schrödinger-like equation [31, 45, 47],

$$
\begin{aligned}
& \rho \rightarrow|\widetilde{\Phi}\rangle=\sum_{m, n=1}^{N} \rho_{m n}(t)\left|E_{m}\right\rangle\left|e_{n}\right\rangle, \\
& i \frac{\partial}{\partial t}|\widetilde{\Phi}\rangle=\mathcal{H}_{T}|\widetilde{\Phi}\rangle
\end{aligned}
$$

where $\rho_{m n}(t)=\left\langle E_{m}|\rho(t)| E_{n}\right\rangle$ is the element of the density matrix. $\left\{\left|E_{n}\right\rangle\right\}$ and $\left\{\left|e_{n}\right\rangle\right\}$ are the time-independent bases for the spin and the ancilla, respectively. $\mathcal{H}_{T}$ is the so-called non-Hermitian effective Hamiltonian. For the considered moving magnetically-driven spin(Eq.(2) ) and the master equation (11), the effective Hamiltonian has the following form,

$$
\mathcal{H}_{T}=H_{K}-H_{K}^{\mathrm{A}}+V-V^{\mathrm{A}}+H_{S}-H_{S}^{\mathrm{A}}+L_{S},
$$

where the superscript A denotes operators of the auxiliary system, which are defined by

$$
\left\langle e_{m}\left|O^{\mathrm{A}}\right| e_{n}\right\rangle=\left\langle E_{n}\left|O^{\dagger}\right| E_{m}\right\rangle,
$$

and $H_{K}^{\mathrm{A}}=H_{K}^{\mathrm{A}}\left(\vec{x}^{\mathrm{A}}\right), \quad V^{\mathrm{A}}=V^{\mathrm{A}}\left(\vec{x}^{\mathrm{A}}\right), \quad H_{S}^{\mathrm{A}}=$ $H_{S}^{\mathrm{A}}\left(\vec{B}\left(\vec{x}^{\mathrm{A}}\right), \vec{S}^{\mathrm{A}}\right), L_{S}$ represents the spin relaxation, its form depends on the coupling of the system to its environment and hence it is a function of the jump operators. For spin relaxation/dissipation (Eq.(3)), we have

$$
L_{S}=i \gamma \sigma^{-} \tau^{-}-\frac{i}{2} \gamma \sigma^{+} \sigma^{-}-\frac{i}{2} \gamma \tau^{+} \tau^{-},
$$

where $\tau^{+}$and $\tau^{-}$are Pauli operators for the auxiliary system. Notice that there is only one term in $L_{S}$ describes the interaction between the quantum system and the ancilla. Now we are in a position to extend the BO approximation from closed to open quantum systems. In order to simplify the derivation and make the formulation more explicit, we define a new six-dimension coordinate by the coordinates of the quantum system and the ancilla as $\vec{r}=\left(\vec{x}, i \vec{x}^{\mathrm{A}}\right)$. With these notations, the effective Hamiltonian can be rewritten as $\mathcal{H}_{T}=H_{K}^{\mathrm{T}}(\vec{r})+V^{\mathrm{T}}(\vec{r})+H_{S}^{\mathrm{T}}(\vec{r})$ with $H_{K}^{\mathrm{T}}(\vec{r})=H_{K}(\vec{x})-H_{K}^{\mathrm{A}}\left(\vec{x}^{\mathrm{A}}\right), V^{\mathrm{T}}(\vec{r})=V(\vec{x})-V^{\mathrm{A}}\left(\vec{x}^{\mathrm{A}}\right)$ and $H_{S}^{\mathrm{T}}(\vec{r})=H_{S}(x)-H_{S}^{\mathrm{A}}\left(x^{\mathrm{A}}\right)+L_{S} \cdot H_{S}^{\mathrm{T}}(\vec{r})$ is the total spin part of the effective Hamiltonian $\mathcal{H}_{T}$. We denote the right eigenfunctions of $H_{S}^{\mathrm{T}}(\vec{r})$ by $\left|\Lambda_{n}^{R}\right\rangle=\left|\Lambda_{n}^{R}(\vec{r})\right\rangle$, $\left(n=1,2, \ldots, N^{2}\right)$ and the left eigenfunctions by $\left\langle\Lambda_{n}^{L}\right|=$ $\left\langle\Lambda_{n}^{L}(\vec{r})\right|$ with the corresponding eigenvalues $E_{n}=E_{n}(\vec{r})$ for a fixed $\vec{r}$. Generally speaking, the total spin part $H_{S}^{\mathrm{T}}$ is not Hermite and the eigenvalues are usually complex. Nevertheless the left and right eigenfunctions still satisfy the orthonormal relation $\left\langle\Lambda_{m}^{L} \mid \Lambda_{n}^{R}\right\rangle=\delta_{m n}$. We should note that when we map the density matrix into a wavefunction of the composite system, degeneracies may be introduced. The occurrence of degeneracies is a signature of the presence of symmetry in the composite system [50]. In the following, we have restricted our discussion on the non-degenerate energy levels.

We can expand the eigenstate of the effective Hamiltonian $\mathcal{H}_{T}$ in terms of $\left\{\left|\Lambda_{n}^{R}\right\rangle\right\}$ as

$$
|\widetilde{\Phi}\rangle=\sum_{k=1}^{N^{2}} \Phi_{n}(\vec{r})\left|\Lambda_{n}^{R}(\vec{r})\right\rangle .
$$

Substituting Eq.(8) into the eigenvalue equation of the effective Hamiltonian $\mathcal{H}_{T}|\widetilde{\Phi}\rangle=E|\widetilde{\Phi}\rangle$, we obtain the following equation for $\Phi_{n}(\vec{r})$,

$$
H^{\mathrm{T}}(n) \Phi_{n}+F^{\mathrm{T}}(n) \Phi_{n}+\sum_{m \neq n} O^{\mathrm{T}}(n, m) \Phi_{m}=E \Phi_{n},
$$

where

$$
\begin{aligned}
& H^{\mathrm{T}}(n)=-\frac{1}{2 M}\left[\nabla_{\vec{r}}-i \vec{A}_{\vec{r}}^{\mathrm{T}}(n)\right]^{2}+V^{\mathrm{T}}(\vec{r})+E_{n}(\vec{r}), \\
& \vec{A}_{\vec{r}}^{\mathrm{T}}(n)=i\left\langle\Lambda_{n}^{L} \mid \nabla_{\vec{r}} \Lambda_{n}^{R}\right\rangle, \\
& F^{\mathrm{T}}(n)=-\frac{1}{2 M} \sum_{m \neq n}\left(\left\langle\Lambda_{n}^{L} \mid \nabla_{\vec{r}} \Lambda_{m}^{R}\right\rangle\left\langle\Lambda_{m}^{L} \mid \nabla_{\vec{r}} \Lambda_{n}^{R}\right\rangle\right), \\
& O^{\mathrm{T}}(n, m)=-\frac{1}{2 M}\left(2\left\langle\Lambda_{n}^{L} \mid \nabla_{\vec{r}} \Lambda_{m}^{R}\right\rangle \nabla_{\vec{r}}+\left\langle\Lambda_{n}^{L} \mid \nabla_{\vec{r}}^{2} \Lambda_{m}^{R}\right\rangle\right)
\end{aligned}
$$

$\nabla_{\vec{r}}$ in the above equations denotes the derivative with respect to the new coordinates $\vec{r}$. We can see from these equations that $\left|\nabla_{\vec{r}} \Lambda_{m}^{R}\right\rangle=0$ for all $m$ may lead $F^{\mathrm{T}}$ and $O^{\mathrm{T}}$ to vanishing, this results in a complete separation of the spin and motion variables in the dynamics, which is quite different from closed systems. It is easy to find one solution that these condition can be met when the dissipation is negligible and the external field is homogeneous. Treat $F^{\mathrm{T}}$ and $O^{\mathrm{T}}$ as perturbations, we can use 
the standard perturbation theory to solve Eq.(9). First we rewrite Eq.(91) as

$$
\left(\mathcal{H}_{0}+\mathcal{W}\right) \Phi=E \Phi
$$

where $\Phi, \mathcal{H}_{0}$ and the perturbation $\mathcal{W}$ are defined as

$$
\begin{gathered}
\Phi=\left[\begin{array}{c}
\Phi_{1} \\
\Phi_{2} \\
\vdots \\
\Phi_{N^{2}}
\end{array}\right], \mathcal{H}_{0}=\left[\begin{array}{cccc}
H^{\mathrm{T}}(1) & 0 & \cdots & 0 \\
0 & H^{\mathrm{T}}(2) & \cdots & 0 \\
\vdots & \vdots & \ddots & \vdots \\
0 & 0 & \cdots & H^{\mathrm{T}}\left(N^{2}\right)
\end{array}\right] \\
\mathcal{W}=\left[\begin{array}{cccc}
F^{\mathrm{T}}(1) & O^{\mathrm{T}}(1,2) & \cdots & O^{\mathrm{T}}\left(1, N^{2}\right) \\
O^{\mathrm{T}}(2,1) & F^{\mathrm{T}}(2) & \cdots & \left.O^{\mathrm{T}}\left(2, N^{2}\right)\right) \\
\vdots & \vdots & \ddots & \vdots \\
O^{\mathrm{T}}\left(N^{2}, 1\right) & O^{\mathrm{T}}\left(N^{2}, 2\right) & \cdots & F^{\mathrm{T}}\left(N^{2}\right)
\end{array}\right]
\end{gathered}
$$

By making use of the time-independent perturbation theory, we can obtain high-order approximations for Eq.(9) or (14). The zeroth order approximation solutions

$$
\begin{gathered}
\widetilde{\Phi}_{\vec{k}_{\vec{r}}}^{[0]}(1)=\left[\begin{array}{c}
\Phi_{\overrightarrow{k_{\vec{r}}}}^{[0]}(1) \\
0 \\
\vdots \\
0
\end{array}\right], \widetilde{\Phi}_{\overrightarrow{k_{\vec{r}}}}^{[0]}(2)=\left[\begin{array}{c}
0 \\
\Phi_{\vec{k}_{\vec{r}}}^{[0]}(2) \\
\vdots \\
0
\end{array}\right], \\
\left.\ldots, \quad \cdots, \quad \begin{array}{c}
0 \\
0 \\
\vdots \\
\Phi_{\overrightarrow{k_{\vec{r}}}}^{[0]}\left(N^{2}\right)
\end{array}\right],
\end{gathered}
$$

are given by the eigenvalue equation

$$
H^{\mathrm{T}}(n) \Phi_{\vec{k}_{\vec{r}}}^{[0]}(n)=E_{\vec{k}_{\vec{r}}}^{[0]}(n) \Phi_{\vec{k}_{\vec{r}}}^{[0]}(n) .
$$

$\vec{k}_{\vec{r}}$ in the above equations represents a wave-vector defined by $\vec{k}_{\vec{r}}=\left(\vec{k}, i \vec{k}^{\mathrm{A}}\right)$. This zeroth order solution indicates that in the six-dimension space, the system and ancilla move in an external potential $V(\vec{r})$ as a whole system and evolve according to the free Hamiltonian $H^{\mathrm{T}}(\vec{r})$. The interaction between the spin and the magnetic field together with the spin relaxation make the two systems feel a complex vector potential $\vec{A}_{\vec{r}}^{\text {T }}$ which would in turn affect the center-of-mass motion of the particle.

From these zeroth order solutions, we can obtain the first order correction as

$$
\begin{aligned}
& E_{\vec{k}_{\vec{r}}}^{[1]}(n)=F^{\mathrm{T}}(n) \\
& \widetilde{\Phi}_{\vec{k}_{\vec{r}}}^{[1]}(n)=\sum_{\substack{n^{\prime} \neq n \\
\vec{k}_{\vec{r}} \neq \vec{k}_{\vec{r}}^{\prime}}} \frac{\left\langle\Phi_{\vec{k}_{\vec{r}}^{\prime}}^{[0]}\left(n^{\prime}\right)\left|O\left(n^{\prime}, n\right)\right| \Phi_{\vec{k}_{\vec{r}}}^{[0]}(n)\right\rangle}{E_{\vec{k}_{\vec{r}}^{\prime}}^{[0]}\left(n^{\prime}\right)-E_{\vec{k}_{\vec{r}}}^{[0]}(n)} \widetilde{\Phi}_{\vec{k}_{\vec{r}}^{\prime}}^{[0]}\left(n^{\prime}\right) .(18)
\end{aligned}
$$

The condition with which we can safely neglect the first order correction is

$$
\left|\frac{\left\langle\Phi_{\vec{k}_{\vec{r}}^{\prime}}^{[0]}\left(n^{\prime}\right)\left|O\left(n^{\prime}, n\right)\right| \Phi_{\overrightarrow{k_{\vec{r}}}}^{[0]}(n)\right\rangle}{E_{\vec{k}_{\vec{r}}^{\prime}}^{[0]}\left(n^{\prime}\right)-E_{\vec{k}_{\vec{r}}}^{[0]}(n)}\right| \ll 1, \vec{k}_{\vec{r}}^{\prime}, n^{\prime} \neq \vec{k}_{\vec{r}}, n .
$$

This is the validity condition for the zeroth order $\mathrm{BO}$ approximation.

In this section, we have extended the $\mathrm{BO}$ approximation to an open system with spin relaxations. The zeroth order approximation was defined as the regime where its effective Hamiltonian can be diagonalized with fixed slowly changing variables. This generalization is available for dissipations in all variables except coordinates (or center-of-mass motion), which we shall study in the next section.

\section{DISSIPATION OF CENTER-OF-MASS MOTION}

We consider a moving particle coupled to a boson bath, the interaction between the particle and the bath can be described by [24]

$$
H_{I}=x \otimes \sum_{k} g_{k}\left(b_{k}+b_{k}^{\dagger}\right),
$$

where $x$ is the coordinate operator for the particle, $b_{k}$ and $b_{k}^{\dagger}$ are the annihilation and creation operators for the bath, respectively. By a standard procedure, we can derive a master equation and write it in the form of Eq.(11), but the dissipation term $\mathcal{L}$ in this case takes

$$
\begin{aligned}
\mathcal{L} \rho & =\gamma_{1}\left(2 x \rho x-\rho x^{2}-x^{2} \rho\right) \\
& +\gamma_{2}(x p \rho+\rho p x-x \rho p-p \rho x) .
\end{aligned}
$$

Define $C=C^{\dagger}=\sqrt{\gamma_{1}} x$ and $D=D^{\dagger}=\sqrt{\gamma_{1}} x-\frac{\gamma_{2}}{\sqrt{\gamma_{1}}} p$, the dissipation term can be rewritten as $\mathcal{L} \rho=C \rho D^{\dagger}+$ $D \rho C^{\dagger}-\frac{1}{2}\left\{D^{\dagger} C, \rho\right\}-\frac{1}{2}\left\{C^{\dagger} D, \rho\right\}$, which can not be arranged in the Lindblad form [51]. Following the same method as that in the last section, we obtain an effective Hamiltonian similar to Eq.(5), but in this section, we replace $L_{S}$ by $L_{C}$ with $L_{C}=\frac{i}{2}\left[2 \gamma_{1} x^{2}-\gamma_{2}(x p+p x)\right]-$ $\frac{i}{2}\left[2 \gamma_{1}\left(x^{\mathrm{A}}\right)^{2}-\gamma_{2}\left(x^{\mathrm{A}} p^{\mathrm{A}}+p^{\mathrm{A}} x^{\mathrm{A}}\right)\right]-i\left[2 \gamma_{1} x x^{\mathrm{A}}-\gamma_{2}\left(x p^{\mathrm{A}}+\right.\right.$ $\left.\left.p x^{\mathrm{A}}\right)\right]$. We choose the eigenfunctions of $H_{S}^{\mathrm{T}}=H_{S}-H_{S}^{\mathrm{A}}$ as the bases, which are a direct product of the eigenfunctions of $H_{S}$ and $H_{S}^{\mathrm{A}}$. Let $H_{S}$ and $H_{S}^{\mathrm{A}}$ have the eigenfunctions $\left|\chi_{m}(x)\right\rangle$ and $\left|\chi_{m}^{\mathrm{A}}\left(x^{\mathrm{A}}\right)\right\rangle$ with the same eigenvalues $\varepsilon_{m}$ for $x=x^{\mathrm{A}}$, we can expand the eigenstate of $\mathcal{H}_{T}$ by $\left\{\left|\Lambda_{m n}\left(x, x^{\mathrm{A}}\right)\right\rangle=\left|\chi_{m}(x)\right\rangle\left|\chi_{n}^{\mathrm{A}}\left(x^{\mathrm{A}}\right)\right\rangle\right\}$ as $|\widetilde{\Phi}\rangle=$ $\sum_{m n} \Phi_{m n}\left|\Lambda_{m n}\right\rangle$. Following the same derivation presented in Sec II we obtain the following equation for $\Phi_{m n}$ as,

$$
\begin{array}{r}
H^{\mathrm{T}}(m n) \Phi_{m n}+F^{\mathrm{T}}(m n) \Phi_{m n}+\sum_{p q \neq m n} O^{\mathrm{T}}(m n, p q) \Phi_{p q} \\
+\sum_{p q \neq m n} L_{C}^{\mathrm{T}}(m n, p q) \Phi_{p q}=E \Phi_{m n} .
\end{array}
$$

where

$$
\begin{aligned}
H^{\mathrm{T}}(m n) & =-\frac{1}{2 M}\left[\nabla_{\vec{r}}-i \vec{A}_{\vec{r}}^{\mathrm{T}}(m n)\right]^{2} \\
& +V^{\mathrm{T}}(\vec{r})+E_{m n}(\vec{r})+L_{C}^{\mathrm{T}}(m n),
\end{aligned}
$$


and

$$
L_{C}^{\mathrm{T}}(m n, p q) \Phi_{p q}=\left\langle\Lambda_{m n}\left|L_{C}\right| \Lambda_{p q}\right\rangle .
$$

When the two subscripts in $L_{C}^{\mathrm{T}}$ are same, we will omit one of them to shorten the notation. $p q \neq m n$ in the summation means $(p, m)$ and $(q, n)$ can not be taken equal simultaneously. Other terms are similar to the results given in Sec II as $F^{\mathrm{T}}(n)=$ $-\frac{1}{2 M} \sum_{p q \neq m n}\left(\left\langle\Lambda_{m n} \mid \nabla_{\vec{r}} \Lambda_{p q}\right\rangle\left\langle\Lambda_{p q} \mid \nabla_{\vec{r}} \Lambda_{m n}\right\rangle\right), O^{\mathrm{T}}(m n, p q)=$ $-\frac{1}{2 M}\left(2\left\langle\Lambda_{m n} \mid \nabla_{\vec{r}} \Lambda_{p q}\right\rangle \nabla_{\vec{r}}+\left\langle\Lambda_{m n} \mid \nabla_{\vec{r}}^{2} \Lambda_{p q}\right\rangle\right)$. We would like to note that because of the bases we choose are uncoupled between the quantum system and ancilla, the Hamiltonian $H^{\mathrm{T}}(m n)$ governing the center-ofmass motion of the system can be factorized as $H^{\mathrm{T}}(m n)=-\frac{1}{2 M}[\nabla-i \vec{A}(m)]^{2}+V+\varepsilon_{m}-\left(-\frac{1}{2 M}\left[\nabla^{\mathrm{A}}-\right.\right.$ $\left.\left.i \vec{A}^{\mathrm{A}}(n)\right]^{2}+V^{\mathrm{A}}+\varepsilon_{n}\right)+L_{C}^{\mathrm{T}}(m n)$ with $\vec{A}(m)=i\left\langle\chi_{m} \mid \nabla \chi_{m}\right\rangle$ and $\vec{A}^{\mathrm{A}}(n)=i\left\langle\chi_{n}^{\mathrm{A}} \mid \nabla^{\mathrm{A}} \chi_{n}^{\mathrm{A}}\right\rangle$, where $\nabla^{\mathrm{A}}$ represents the derivative with respect to $\vec{x}^{\mathrm{A}}$. This means that the interaction between the system and the magnetic field makes the system and the ancilla feel vector potentials $\vec{A}(m)$ and $\vec{A}^{\mathrm{A}}(n)$, respectively. DissCOM induces a correction $L_{C}^{\mathrm{T}}(m n)$ to the system via the interaction between the system and ancilla in $H^{\mathrm{T}}(m n)$. In the same way, namely treat the terms $F^{\mathrm{T}}, O^{\mathrm{T}}$ and $L_{C}^{\mathrm{T}}(m n, p q),(m n \neq p q)$ as perturbations, we obtain the validity condition for the zero order $\mathrm{BO}$ approximation

$$
\begin{array}{r}
\left|\frac{\left\langle\Phi_{\vec{k}_{\vec{r}}^{\prime}}^{[0]}(p q)\left|\left(O^{\mathrm{T}}(p q, m n)+L_{C}^{\mathrm{T}}(p q, m n)\right)\right| \Phi_{\vec{k}_{\vec{r}}}^{[0]}(m n)\right\rangle}{E_{\vec{k}_{\vec{r}}^{\prime}}^{[0]}(p q)-E_{\vec{k}_{\vec{r}}}^{[0]}(m n)}\right| \ll 1, \\
m n, \vec{k}_{\vec{r}} \neq p q, \vec{k}_{\vec{r}}^{\prime}, \quad(23)
\end{array}
$$

where

$$
L_{C}^{\mathrm{T}}(m n, p q) \Phi_{p q}=\left\langle\Lambda_{m n}\left|L_{C}\right| \Lambda_{p q}\right\rangle,
$$

and

$$
\begin{aligned}
L_{C} & =\frac{i}{2}\left[2 \gamma_{1} x^{2}-\gamma_{2}(x p+p x)\right] \\
& -\frac{i}{2}\left[2 \gamma_{1}\left(x^{\mathrm{A}}\right)^{2}-\gamma_{2}\left(x^{\mathrm{A}} p^{\mathrm{A}}+p^{\mathrm{A}} x^{\mathrm{A}}\right)\right]
\end{aligned}
$$

$$
-i\left[2 \gamma_{1} x x^{\mathrm{A}}-\gamma_{2}\left(x p^{\mathrm{A}}+p x^{\mathrm{A}}\right)\right] .
$$

From Eqs.221) and (23) we can find that the zeroorder Hamiltonian $H^{\mathrm{T}}$ only contains the diagonal elements of $L_{C}^{\mathrm{T}}$, while the perturbation matrix includes the spin part $O^{\mathrm{T}}, F^{\mathrm{T}}$, and the spatially dependent term $L_{C}^{\mathrm{T}}(m n, p q),(m n \neq p q)$. Recall that the main concept of $\mathrm{BO}$ approximation in our case is to separate the spin variables from the spatial one (i.e., the fast and slow variables). Due to the dissipation represented by $L_{C}^{\mathrm{T}}$, this separation of those variables becomes difficult, resulting in the violation of $\mathrm{BO}$ approximation. We will show in the next section that this is different for the case of spin relaxation, because the spin relaxation is coordinate independent.

\section{EXAMPLE}

As an example, in this section we consider a neutron moving in a static helical magnetic field,

$$
\vec{B}=\vec{B}(z)=B\left(\sin \theta \cos \frac{2 \pi z}{L}, \sin \theta \sin \frac{2 \pi z}{L}, \cos \theta\right)
$$

The Hamiltonian for such a system is

$$
H=H(z)=\frac{\vec{p}^{2}}{2 M}+\mu \vec{B} \cdot \vec{\sigma}=H_{K}+H_{S}
$$

For fixed but arbitrary $z$, the interaction Hamiltonian $H_{S}$ has the eigenfunctions

$$
\begin{aligned}
& \left|\chi_{1}(z)\right\rangle=\left(\begin{array}{c}
\cos \frac{\theta}{2} \exp \left(-i \frac{2 \pi z}{L}\right) \\
\sin \frac{\theta}{2}
\end{array}\right) \\
& \left|\chi_{2}(z)\right\rangle=\left(\begin{array}{c}
\sin \frac{\theta}{2} \exp \left(-i \frac{2 \pi z}{L}\right) \\
-\cos \frac{\theta}{2}
\end{array}\right),
\end{aligned}
$$

and corresponding eigenvalues $\varepsilon_{1,2}= \pm \mu B$. This system is the same as that studied in Ref.[22]. Taken the spin relaxation into account (see Eq.(3)), the effective Hamiltonian related to the spin reads

$$
H_{S}^{\mathrm{T}}\left(\varphi, \varphi^{\mathrm{A}}\right)=\mu B\left(\begin{array}{cccc}
-i g & -\sin \theta e^{i \varphi^{\mathrm{A}}} & \sin \theta e^{-i \varphi} & 0 \\
-\sin \theta e^{-i \varphi^{\mathrm{A}}} & 2 \cos \theta-\frac{1}{2} i g & 0 & \sin \theta e^{-i \varphi} \\
\sin \theta e^{i \varphi} & 0 & -2 \cos \theta-\frac{1}{2} i g & -\sin \theta e^{i \varphi^{\mathrm{A}}} \\
i g & \sin \theta e^{i \varphi} & -\sin \theta e^{-i \varphi^{\mathrm{A}}} & 0
\end{array}\right)
$$

where $\varphi=\frac{2 \pi z}{L}$ and $\varphi^{\mathrm{A}}=\frac{2 \pi z^{\mathrm{A}}}{L}$. The rescaled coupling constant is defined by $g=\frac{\gamma}{\mu B}$ and therefore it is dimensionless. In the following, we set $\theta=\frac{\pi}{2}$. The eigenvalue
$E_{j}$ of $H_{S}^{\mathrm{T}}\left(\phi, \phi^{\mathrm{A}}\right)$ in this case are given by (in units of $\mu B$ )

$$
E_{1}=-\frac{1}{2} g i,
$$




$$
\begin{array}{r}
E_{j}^{3}+\frac{3}{2} g i E_{j}^{2}-\frac{1}{2}\left(8+g^{2}\right) E_{j}+2 g i\left(e^{-i\left(\varphi-\varphi^{\mathrm{A}}\right)}-1\right)=0, \\
(j=2,3,4),
\end{array}
$$

the corresponding right eigenstates are

$$
\left|R_{j}\right\rangle=\left(\begin{array}{c}
A_{j} \\
B_{j} \\
C_{j} \\
D_{j}
\end{array}\right),
$$

while the left eigenstates read

$$
\left\langle L_{j}\right|=\frac{1}{N_{j}}\left(a_{j}, b_{j}, c_{j}, d_{j}\right) .
$$

Here

$$
N_{j}=\left(A_{j} a_{j}+B_{j} b_{j}+C_{j} c_{j}+D_{j} d_{j}\right) .
$$

For $j=1$,

$$
\begin{aligned}
& A_{1}=D_{1}=a_{1}=d_{1}=0, \\
& B_{1}=e^{-i \varphi^{\mathrm{A}}}, \quad C_{1}=e^{i \varphi} \\
& b_{1}=e^{i \varphi^{\mathrm{A}}}, \quad c_{1}=e^{-i \varphi}
\end{aligned}
$$

and for $j=2,3,4$,

$$
\begin{aligned}
& A_{j}=4-g i E_{j}-2 E_{j}^{2}, \\
& B_{j}=-2 e^{-i \varphi} g i+2 e^{-i \varphi^{\mathrm{A}}} E_{j}, \\
& C_{j}=2 e^{i \varphi^{\mathrm{A}}} g i-2 e^{i \varphi} E_{j}, \\
& D_{j}=4 e^{i\left(\varphi-\varphi^{\mathrm{A}}\right)}+g\left(g-2 i E_{j}\right), \\
& a_{j}=e^{i\left(\varphi-\varphi^{\mathrm{A}}\right)}\left(4-g i E_{j}-2 E_{j}^{2}\right), \\
& b_{j}=2 e^{i \varphi} E_{j}, \\
& c_{j}=-2 e^{-i \varphi^{\mathrm{A}}} E_{j}, \\
& d_{j}=4 .
\end{aligned}
$$

We can see from the eigenvalues and eigenstates that only when $\varphi=\varphi^{\mathrm{A}}$, i.e. $z=z^{\mathrm{A}}$, there exists a steady state for the quantum system [47], which is independent of initial state. This is exactly the situation we will with in the following discussion, in which we will discuss the population transfer among the internal states for the quantum system. Initially, the spin of the neutron is prepared in the state $\left|+\frac{1}{2}\right\rangle$, we manipulate the neutron moved from $z=0$ to $z=L$ in a time interval $T$ and calculate the polarization of the neutron along the $z$ axis at time $T$. The polarization of the neutron along $z$ axis at time $T$ with different rescaled dissipation rate is plotted in Fig 1. Several features can be found from these figures. (1) When $g \rightarrow 0$, the relation between the polarization along $z$ axis and the duration $T$ is a cosine function. This coincides with the results given in Ref.[22], meaning that our description can return back to the results for closed systems. (2) As $g$ increases, the relation between the polarization along $z$ axis and duration $T$ is also an oscillating

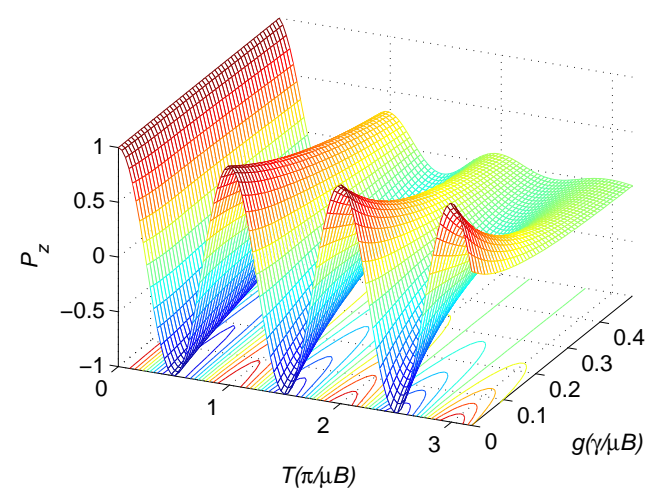

FIG. 1: The polarization of the neutron along the $z$ axis as functions of the duration $T$ and the rescaled dissipation rate $g$. The time $T$ is plotted in units of $\pi / \mu B$. Initially the spin is in the state $\left|+\frac{1}{2}\right\rangle$, i.e. $P_{z}=1$.

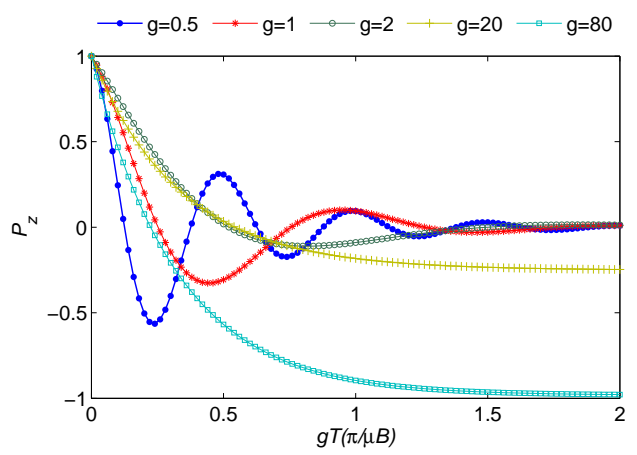

FIG. 2: (Color Online)The polarization of the neutron along the $z$ axis for different $g$ as a function of $g T$ (in units of $\pi / \mu B$ ).

function, but the amplitude of the oscillation decreases with the interval $T$. (3) With $g \rightarrow 0.5$ and $T \rightarrow 3(\pi / \mu B)$, the polarization along $z$ axis reaches zero, arrives at a steady value. These features all result from the competition between the spin-field coupling represented by $H_{S}$ and the dissipation $\mathcal{L}$. When $T$ and $g$ are large enough, the accumulation of the dissipation leads the spin of the neutron into the steady state, a result of the balance between the spin-field coupling $H_{S}$ and the dissipation. We plot the polarization along $z$ axis as a function of $g T$ for different $g$ in Fig.2. We can see from the figure that when $g \rightarrow \infty, P_{z}$ sharply drops into -1, i.e. the spin dissipates into the state $\left|-\frac{1}{2}\right\rangle$. This can be easily understood as follows. When $g \rightarrow \infty$, the spin-field coupling $H_{S}$ can be neglected, namely the dissipation dominates the dynamics of the spin, which makes the spin relax to its ground state $\left|-\frac{1}{2}\right\rangle$.

Finally, we discuss the validity condition for the BO approximation. For the example under discussion, if we do not consider the dissipation, i.e., the system is closed, the validity condition given in this paper returns to the validity condition derived in Ref. 22], this means that the BO approximation for open systems defined in this 


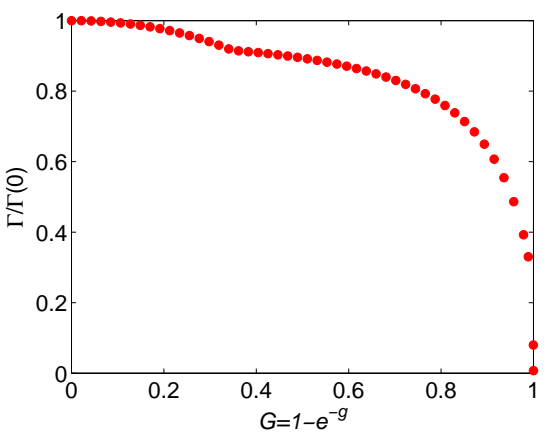

FIG. 3: Validity measure $\Gamma(g)$ as a function of the rescaled dissipation rate $g$. Other parameters in the figure are set to satisfy $\frac{1}{\mu B M^{2} L}=10^{-6}, \frac{k_{z}}{\mu B M L}=2 \times 10^{-4}$. The results have been normalized in units of $\Gamma(g=0)$.

paper can back to closed systems. In order to see the effect of dissipation on the validity condition, we recall that without any dissipation, the validity condition for this example is 22 ]

$$
\frac{1}{\mu B M^{2} L} \ll 1, \frac{k_{z}}{\mu B M L} \ll 1,
$$

where $k_{z}=\frac{v_{z}}{M}$. Namely, when the magnetic field is homogeneous and strong enough as well as the velocity of the neutron along the $z$ axis is very small, the BO approximation is a good approximation. In the following, we study how the dissipation affects the validity condition for the open system. To show the dependence of the validity condition on the dissipation rate, we define the following function with max taken over all $m$ and $n$ except $m=n$,

$$
\Gamma(g)=\max \left\{\left|\frac{\left\langle\Phi_{\vec{k}_{\vec{r}}^{\prime}}^{[0]}(n)|O(n, m)| \Phi_{\vec{k}_{\vec{r}}}^{[0]}(m)\right\rangle}{E_{\vec{k}_{\vec{r}}^{\prime}}^{[0]}(n)-E_{\overrightarrow{k_{\vec{r}}}}^{[0]}(m)}\right|\right\},
$$

to characterize the violation of the BO approximation. The numerical results of $\Gamma(g)$ on $g$ is shown in Fig. 3 , In this figure, we have set all parameters except $g$ to satisfy the BO approximation condition for closed system given in Ref. 22] (e.g., $\frac{1}{\mu B M^{2} L}=10^{-6}, \frac{k_{z}}{\mu B M L}=2 \times 10^{-4}$ ), and have normalized the results with respect to $\Gamma(g=0)$, i.e. all the values in the figure are rescaled by $\Gamma(0)$. We can find that as $g$ increases, $\Gamma(g)$ decreases. This result tells us that if the closed system satisfies the BO approximation condition, the corresponding open system satisfies that condition too. Two points are worth addressing. (1) This conclusion depends sharply on the dissipation, for a given dissipation, we should check the validation condition case by case, and (2) the dissipation benefits the BO approximation in this example, this can be understood as follows. In this example, the BO approximation for open systems is defined as the possibility to separate the spin and spatial variables, the dissipation occurs only for the spin (slowly changed) variable and it is coordinate independent, so it is reasonable that the dissipation benefits the BO approximation. Similar conclusions can be found in the adiabatic approximation in open systems, see Ref. 37, 45, 47].

\section{CONCLUSION AND DISCUSSION}

In this paper, we have extended BO approximation from closed to open systems by the effective Hamiltonian approach. Two types of dissipation are considered, the conditions under which the BO approximation holds are given and discussed. When the spin relaxation/dissipation is considered, the center-of-mass of the system feels a complex vector potential depending on the interaction between the spin and the magnetic field as well as the dissipation rate. The zeroth order BO approximation was defined as the regime in which its effective Hamiltonian can be diagonalized with fixed slowly changing variables. When there is only DissCOM, the vector potential can be factorized into two parts, which are for the system and ancilla, respectively. The DissCOM introduces a correction to the Hamiltonian for the center-of-mass motion and then modifies the dynamics of the system. An example with spin relaxation/dissipation has been presented and discussed.

We thank Zheng-Yuan Xue for help. This work is supported by NSF of China under grant No. 10775023.
[1] M. Born and V. Fork, Z. Phys. 51, 165 (1928).

[2] M. Born and R. Oppenheimer, Ann. Phys. 84, 457 (1927).

[3] K.-P. Marzlin and B. C. Sanders, Phys. Rev. Lett. 93, 160408 (2004) (e-print: quan-ph/0404022); S. Duki, H. Mathur, and O. Narayan, Phys. Rev. Lett. 97, 128901 (2006); J. Ma, Yongping Zhang, Enge Wang and Biao Wu, Phys. Rev. Lett. 97, 128902 (2006); K.-P. Marzlin and B. C. Sanders, Phys. Rev. Lett. 97, 128093 (2006).

[4] Zhaoyan Wu and Hui Yang, Phys. Rev. A 72, 012114
(2005) (e-print: quan-ph/0410118).

[5] D. M. Tong, K. Singh, L. C. Kwek, and C. H. Oh, Phys. Rev. Lett. 95, 110407 (2005) (e-print: quan-ph/0509073).

[6] D. M. Tong, K. Singh, L. C. Kwek, and C. H. Oh, Phys. Rev. Lett. 98, 150402 (2007) (e-print: arXiv:0704.1184).

[7] R. MacKenzie, A. Morin-Duchesne, H. Paquette, and J. Pinel, Phys. Rev. A 76, 044102 (2007) (e-print: arXiv:0706.0885).

[8] M. Y. Ye, X. F. Zhou, Y. S. Zhang, and G. C. Guo, Phys. 
Lett. A 368, 18 (2007) (e-print: arXiv: 0801.0521).

[9] Yan Zhao, Phys. Rev. A 77, 032109 (2008).

[10] Jiangfeng Du, Lingzhi $\mathrm{Hu}$, Ya Wang, Jianda Wu, Meisheng Zhao, and Dieter Suter, Phys. Rev. Lett. 101, 060403 (2008) (e-print: arXiv: 08010361).

[11] K. Fujikawa, Phys. Rev. D 77, 045006 (2008) (e-print: arXiv: 0710.5341).

[12] D. Comparat, Phys. Rev. A 80, 012106 (2009).

[13] M. V. Berry, Proc. R. Soc. London A 392, 45 (1984).

[14] Y. Aharonov and J. Anandan, Phys. Rev. Lett. 58, 1593 (1987).

[15] E. Sjöqvist, A. K. Pati, A. Ekert, J. S. Anandan, M. Ericsson, D. K. L. Oi and V. Vedral, Phys. Rev. Lett. 85, 2845 (2000) (e-print: quan-ph/0005072).

[16] D. M. Tong, E. Sjöqvist, L. C. Kwek, and C. H. Oh, Phys. Rev. Lett. 93, 080405 (2004) (e-print: quan-ph/0405092).

[17] A. T. Rezakhani, and P. Zanardi, Phys. Rev. A 73, 052117 (2006) (e-print: quan-ph/0512250).

[18] Z. S. Wang, Chunfeng Wu, X. L. Feng, L. C. Kwek, C. H. Lai, and C. H. Oh, Phys. Rev. A 75, 024102 (2007).

[19] W. L. Yang and J. L. Chen Phys. Rev. A 75, 024101 (2007) (e-print: arXiv: 0706.2063).

[20] D. Møller, L. B. Madsen, and K. Mølmer, Phys. Rev. A 77, 022306 (2008)(e-print: arXiv: 0710.0450).

[21] X. L. Huang and X. X. Yi, Europhysics Letters 82, 50001 (2008)(e-print: arXiv: 0811.1071).

[22] Chang-Pu Sun and Mo-Lin Ge, Phys. Rev. D 41, 1349 (1990).

[23] L. S. Cederbaum, J. Chem. Phys., 128, 124101 (2008).

[24] C. W. Gardiner, Quantum Noise, (New York: Springer, 2000).

[25] L. M. Arévalo-Aguilar and H. Moya-Cessa, Quantum Semiclassic. Opt. 10671 (1998).

[26] A. B. Klimov and J. L. Romero, J. Opt. B, 5, S316 (2003);

[27] H. Nakazato, Y. Hida, K. Yuasa, B. Militello, A. Napoli, and A. Messina, Phys. Rev. A 74, 062113 (2006).

[28] J. Yang, Y. D. Zhang, and Z. B. Chen, Phys. Rev. A 67, 024101 (2003).

[29] X. X. Yi, C. Li, and J. C. Su Phys. Rev. A 62, 013819 (2000)(e-print:q uan-ph/0003039).

[30] H. J. Briegel and B. G. Englert, Phys. Rev. A 47, 3311 (1993).

[31] X. X. Yi and S. X. Yu, J. Opt. B, 3, 372 (2001). (e-print: quan-ph: 0008090).
[32] H. P. Breuer and F. Petruccione, The Theory of Open Quantum Systems (Oxford University Press, Oxford 2002).

[33] H. P. Breuer, J. Gemmer and M. Michel, Phys. Rev. E 73, 016139 (2006)(e-print: quan-ph/0510177).

[34] H. P. Breuer, Phys. Rev. A 75, 022103 (2007) (e-print: quan-ph/0611208).

[35] B. Vacchini, Phys. Rev. A 78, 022112 (2008) (e-print: arXiv: 0805.0561).

[36] S. Maniscalco, Phys. Rev. A 75, 062103 (2007).

[37] A. Shabani and D. A. Lidar, Phys. Rev. A 71, 020101(R) (2005) (e-print quan-ph/0404077).

[38] S. Maniscalco and F. Petruccione, Phys. Rev. A 73, 012111 (2006) (e-print: quan-ph/0509208).

[39] X. L. Huang, J. Nie, J. Chen and X. X. Yi, Phys. Scr. 78025001 (2008).

[40] H. P. Breuer and B. Vacchini, Phys. Rev. Lett. 101, 140402 (2008) (e-print: arXiv: 0809.1501).

[41] M. S. Sarandy and D. A. Lidar, Phys. Rev. A 71, 012331 (2005) (e-print: quan-ph/0404147).

[42] A. Fleischer and N. Moiseyev, Phys. Rev. A 72, 032103 (2005).

[43] P. Thunström, J. Åberg, and E. Sjöqvist, Phys. Rev. A 72, 022328 (2005) (e-print: quan-ph/0504046).

[44] M. J. O'Hara and D. P. O'Leary, Phys. Rev. A 77, 042319 (2008).

[45] X. X. Yi, D. M. Tong, L. C. Kwek and C. H. Oh, J. Phys. B 40, 281 (2007). (e-print: quan-ph: 0606203).

[46] D. M. Tong, X. X. Yi, X. J. Fan, L. C. Kwek, and C. H. Oh, Phys. Lett. A 372, 2364 (2008).

[47] X. L. Huang, X. X. Yi, C. F. Wu, X. L. Feng, S. X. Yu, and C. H. Oh, Phys. Rev. A 78, 062114 (2008). (e-print: arXiv: 0810.2913).

[48] G. Lindblad, Commun. Math. Phys. 48, 119 (1976).

[49] M. O. Scully and M. S. Zubairy Quantum Optics (Cambridge University Press, Cambridge 1997); D. F. Walls and G. J. Milburn Quantum Optics (Springer, Berlin,1994), M. Schlosshauer, Decoherence and the Quantum-to-Classical Transition, 1st ed. (Springer, Berlin, Heidelberg, 2007).

[50] B. A. Tay and T. Petrosky, Phys. Rev. A 76, 042102 (2007).

[51] H. P. Breuer, Phys. Rev. A 70, 012106 (2004) (e-print: quan-ph/0403117). 